\title{
Electromigration of Single-Layer Clusters
}

\author{
O. Pierre-Louis ${ }^{* \dagger}$ and T. L. Einstein ${ }^{\dagger}$ \\ ${ }^{*}$ LSP Grephe, CNRS, UJF-Grenoble 1, BP87, F38402 Saint Martin d'Hères, France \\ ${ }^{\dagger}$ Department of Physics, University of Maryland, College Park, MD 20742-4111
}

\begin{abstract}
Single-layer atom or vacancy clusters in the presence of electromigration are studied theoretically assuming an isotropic medium. A variety of distinctive behaviors distinguish the response in the three standard limiting cases of periphery diffusion (PD), terrace diffusion (TD), and evaporationcondensation (EC). A general model provides power laws describing the size dependence of the drift velocity in these limits, consistent with established results in the case of $\mathrm{PD}$. The validity of the widely used quasistatic limit is calculated. Atom and vacancy clusters drift in opposite directions in the PD limit but in the same direction otherwise. In absence of PD, linear stability analysis reveals a new type of morphological instability, not leading to island break-down. For strong electromigration, Monte Carlo simulations show that clusters then destabilize into slits, in contrast to splitting in the PD limit. Electromigration affects the diffusion coefficient of the cluster and morphological fluctuations, the latter diverging at the instability threshold. An instrinsic attachment-detachment bias displays the same scaling signature as PD in the drift velocity.
\end{abstract}

olivier.pierre-louis@ujf-grenoble.fr; einstein@physics.umd.edu

\section{INTRODUCTION}

Recognized as a key source of size limitation in electrical devices, surface electromigration has important consequences for surface morphology. Latyshev et al. 1 discovered that it can induce step bunching on $\mathrm{Si}(111)$ vicinal surfaces. How electromigration affects more complex surface structures is still poorly understood. In this paper we explore the effect of electromigration of atoms or vacancies on single-layer clusters. Responses of these clusters to electromigration share similarities with void behavior in metallic electric lines and with electromigratipn of oxygen disordered domains in $\mathrm{YBaCuO}$ thin films 3 and lies in the active area of driven diffusive systems 5

In addition to the intrinsic interest in how island properties are affected by the symmetry-breaking electromigration force, we will point out further mesoscopic ways to address current controversies about the dominant mechanism of mass transport. 6 Moreover, these properties are needed to model surfaces undergoing electromigration at larger scales. For example, electromigrationinduced coalescence models should use non-equilibrium diffusion constants and steady-state drift velocities.

In the presence of an electric current, the electromigration force is usually described as $\mathbf{F}=z^{*} e \mathbf{E}$, where $e$ is the magnitude of the electron charge and $z^{*}$ is an effective valence, generally non-integer, which takes into account both the electrostatic interaction between the electric field $\mathbf{E}$ and the charge distribution on the affected atoms ("direct" force) and the frictional force resulting from the transfer of momentum from the charge carriers to these atoms ("wind" force). The long-standing controversies regarding bulk electromigration highlight the complexity of microscopic calculations, and particularly how conduction electrons may screen the direct charge of the atoms. Non-trivial aspects of the flow of the electron cloud about the diffusion path might also contribute to the direct effect on the surface. 8 For metals, the electromigration effect is primarily due to wind force; calculated values include $z^{*} \approx-30$ for $\mathrm{Al}$ on semiinfinite jellium 9 and $z^{*} \approx-21$ for $\mathrm{Cu}$ on $\mathrm{Cu}\{111\} .10$ (Near a step edge, one can expect quantitative but not order-of-magnitude changes in $z^{*}$; e.g. for an atom in a close-packed step edge on $\operatorname{Al}\{001\}, z^{*} \approx-4311$ ) For semiconductors, both forces are small, with a resultant $\left|z^{*}\right| \approx 0.001-0.112$ A characteristic length can be associated with electromigration: $\xi \equiv k_{B} T / F$. In typical experimental conditions, $\xi \sim 10^{8}$ atomic spacings for $\mathrm{Si}$ and $\xi \sim 10^{5}$ for metals. We base our study of the equilibrium behavior of these islands on the framework of the continuum theory of Khare et al.13 We consider electromigration to be a perturbation inducing a macroscopic current, as done previously in Refs. 2 and 14. This approximation is justified because $\xi$ is much larger than the atomic spacing. The adatom flux on terraces is then equal to $c\langle v\rangle$. The mean drift velocity $\langle v\rangle$ of an adatom due to electromigration is calculated from the Einstein relation:

$$
\langle v\rangle=D \frac{F}{k_{B} T}=\frac{D}{\xi} .
$$

Single-vacancy motion may dominate over atom motion in the mass transport on some surfaces. (There is evidence of this on $\mathrm{Cu}\{001\} .15$ (16) In that case one must use an effective charge $z^{*}$ appropriate for vacancies and reverse the signs of step curvatures in computing restoring forces due to line tension. The resulting modifications are relatively straightforward but tend to muddle the subsequent descriptions. Accordingly, we do not explicitly consider vacancy transport below.

In the notation of Ref. 13, there are three limiting mass transport modes: periphery diffusion (PD), terrace diffusion (TD), and (two-dimensional) evaporationcondensation (EC) or attachment-detachment (see Fig. 1). In particular, there is a distinctive size depen- 
dence of the tracer diffusion constant $D_{c}^{e q}$ of a cluster at thermodynamic equilibrium, with characteristic exponent $\alpha=3,2$, and 1 , respectively:

$$
D_{c}^{e q} \sim R_{0}^{-\alpha}
$$

where $R_{0}$ is its average radius. Intermediate values can also be obtained in restricted regions of parameter space as one crosses over from one limiting regime to another. As a first qualitative approach, the mean cluster drift velocity should be given by the Einstein relation in a way very similar to the drift velocity of one adatom (Eq. (11)): it is equal to the cluster mobility (or equilibrium diffusion constant $D_{c}^{e q}$ ) time the force exerted on the whole cluster $\bar{F}=\pi R_{0}^{2} F / a^{2}$, and divided by $k_{B} T$ :

$$
|\bar{V}| \approx D_{c}^{e q} \frac{\bar{F}}{k_{B} T}=D_{c}^{e q} \frac{\pi R_{0}^{2}}{a^{2} \xi} \sim R_{0}^{2-\alpha} .
$$

This scaling law will be confirmed later. The intermediate expressions are in fact exact for models in which atoms approach and leave the island only from the lower edge (one-sided step), or in the PD regime. (In the more general case, some subtleties arise.)

We further investigate the shape changes associated with steady states. Circular steady states are found in the PD and TD regimes. But when attachment and detachment of atoms to the steps is not instantaneous, the cluster elongates. This can be understood by analogy to the Bernoulli effect. Stability of these steady states is studied. Besides the expected splitting of clusters for $\mathrm{PD}$, we find another morphological instability in the TD case. Monte Carlo simulations indicate that it leads to slit formation. Fluctuations and the non-equilibrium diffusion constant are also calculated within the framework of a Langevin model, and appear to be corrected by a bias term proportional to $1 / \xi^{2}$ for weak electromigration.

\section{MODEL}

Our formal approach is inspired by a previous examination of non-equilibrium step meandering on vicinal surfaces.17 For simplicity and to avoid distracting complications, we take all step and terrace properties to be isotropic. We forbid adsorption onto or desorption from the terrace. Evolution of the mobile-atom concentration on terraces is then given by the following conservation law:

$$
\begin{aligned}
\partial_{t} c & =-\nabla \cdot \mathbf{J}, \\
\mathbf{J} & \equiv-D \nabla c+\frac{D}{\xi} c \hat{\mathbf{x}}+\mathbf{q} .
\end{aligned}
$$

$\mathbf{J}$ is the mass flux on terraces, and $D$ is likewise the adatom diffusion constant on terraces. The correlations of the conserved noise $\mathbf{q}$ are given below. In the widely used quasistatic limit, one considers that the adatom concentration reaches a steady state on time scales much smaller than that of step motion. Then

$$
\nabla \cdot \mathbf{J}=0
$$

has to be solved on terraces instead of Eq.(4). Adatom electromigration is taken to be unaffected by the presence of steps; thus, $\xi$ is uniform. Atom exchange between the step and the 2D "gas" on the terrace is proportional to the deviation from equilibrium, expressed in the linear kinetic relation:

$$
\hat{\mathbf{n}} \cdot \mathbf{J}_{ \pm}=\mp \nu_{ \pm}\left(c-c_{e q}-\eta_{ \pm}\right) .
$$

In our notation, + or - denote the lower or the upper side, respectively, of the step forming the boundary of the island. Thus, for atom islands, + is the exterior while for vacancy islands it is the interior. The unit vector $\hat{\mathbf{n}}$ normal to the step points toward the + side. The kinetic coefficients $\nu_{ \pm}$describe attachment and detachment at the two sides of the step edge, and $\eta_{ \pm}$are non-conserved noises. Attachment lengths are defined by $d_{ \pm} \equiv D / \nu_{ \pm}$; the larger $d_{ \pm}$, the smaller the chance that an atom will detach from or attach to the step. When $d_{ \pm}$are small, the dynamics are diffusion limited (TD). For large but finite $d_{ \pm}$, atoms attach only after a large number of trials, and dynamics are thus limited by attachment and detachment (EC). In the limit that $d_{-} \rightarrow \infty$, the model reduces to an exterior (or interior) model for atom (or vacancy) islands, because atom exchange occurs on only one (viz., the lower) side of the step. The equilibrium concentration $c_{e q}$ must include corrections due to boundary curvature, as given by the Gibbs-Thomson relation

$$
c_{e q}=c_{e q}^{0} \exp (\Gamma \kappa)
$$

where $c_{e q}^{0}$ is the equilibrium concentration in the vinicity of a traight step, $\kappa$ is the step curvature (counted positive for a convex step cf. Eq. (22)), and $\Gamma$ is the capillary length:

$$
\Gamma=a^{2} \tilde{\beta} / k_{B} T
$$

The step stiffness $\tilde{\beta}$ is taken to be isotropic, as noted above; $a$ is the lattice constant, and $T$ is the temperature. Finally, the normal velocity $V_{n}$ (i.e., along $\hat{\mathbf{n}}$ ) of the step is given by mass conservation

$$
V_{n}=a^{2} \hat{\mathbf{n}} \cdot\left(\left.\mathbf{J}\right|_{+}-\left.\mathbf{J}\right|_{-}\right)-\partial_{s} J_{s t}
$$

where $s$ is the arclength along the step. We consider the low-concentration limit, where $c \ll 1 / a^{2}$, i.e. the atom concentration is taken to be much lower than in the bulk. Hence, the second term, representing advection, in $\left.\mathbf{J}\right|_{ \pm}=-D \nabla c_{ \pm}+\hat{\mathbf{n}} V_{n} c_{ \pm}$is neglected. $J_{s t}$ is the mass flux along the step. In addition to the usual relaxation term related to chemical potential gradient, electromigration induces a new term $D_{L} F_{s t} n_{y} / k_{B} T=D_{L} n_{y} / \xi_{s t}$, with $\xi_{s t} \equiv k_{B} T / F_{s t} . F_{s t}$ is the force exerted on mobile atoms 
at the step edge, and $n_{y}$ is the $y$ component of $\hat{\mathbf{n}}$. Thus, we have

$$
J_{s t}=a \frac{D_{L}}{\xi_{s t}} \partial_{s}[R(\theta) \cos (\theta)]-a D_{L} \partial_{s}(\Gamma \kappa)-q_{s t},
$$

where $q_{s t}$ is a Langevin force. $R(\theta)$ is the distance from the center of mass of the cluster, and $\theta$ is the polar angle with respect to the $x$ axis (see Fig.1). The macroscopic step diffusion constant could be defined as $D_{L} \equiv a^{2} c_{s t} D_{s t}$, where $c_{s t}$ is concentration of mobile edge-atoms, and $D_{s t}$ is the diffusion constant for motion of these atoms along the step, or using the Kubo formula.18 In the following, we will take $D_{L}$ to be uniform along the step and constant in time.

In a local thermodynamical equilibrium approximation, noise correlations are written

$$
\begin{array}{r}
\left\langle q_{i}(x, y, t) q_{j}\left(x^{\prime}, y^{\prime}, t^{\prime}\right)\right\rangle=2 D c(x, y, t) \delta, \\
\left\langle\eta_{ \pm}(s, t) \eta_{ \pm}\left(s^{\prime}, t^{\prime}\right)\right\rangle=\frac{\left.2 c(s, t)\right|_{ \pm}}{\nu_{ \pm}} \delta\left(s-s^{\prime}\right) \delta\left(t-t^{\prime}\right), \\
\left\langle q_{s t}(s, t) q_{s t}\left(s^{\prime}, t^{\prime}\right)\right\rangle=\frac{a^{3} D_{L}}{\pi} \delta\left(s-s^{\prime}\right) \delta\left(t-t^{\prime}\right),
\end{array}
$$

where $\delta \equiv \delta\left(x-x^{\prime}\right) \delta\left(y-y^{\prime}\right) \delta\left(t-t^{\prime}\right) \delta_{i j}$, and $\left.c(s, t)\right|_{ \pm}$is the adatom concentration on the terraces in the vicinity of the island edge.

In describing the fluctuations of these islands, we consider only the case in which overhangs can be ignored. Thus, the periphery can be described in polar coordinates by $R(\theta)$. For small fluctuations from the circular, $R(\theta)=R_{0}+\rho(\theta)$. The Fourier transform of $\rho$ is given by

$$
\rho_{n \omega}=\int_{0}^{2 \pi} \frac{d \theta}{2 \pi} \int_{-\infty}^{+\infty} \frac{d t}{2 \pi} \rho(\theta, t) e^{-i n \theta-i \omega t} .
$$

The equilibrium properties of such clusters (for $F=0$ ) were calculated in detail by Khare et al. 13 They are able to evaluate the equilibrium diffusion constant under general conditions. Equipartition of energy provides the static spectrum: 19

$$
\left\langle\left|\rho_{n}\right|^{2}\right\rangle_{\mathrm{eq}}=\frac{k_{B} T R_{0}}{2 \pi\left(n^{2}-1\right) \tilde{\beta}} .
$$

The divergence of $\left\langle\left|\rho_{1}\right|^{2}\right\rangle_{\text {eq }}$ reflects the absence of an energy cost to translate the whole island: this is a "Goldstone mode." The mode $n=0$ is forbidden due to area conservation for PD dynamics. But generally in the presence of non-conserved dynamics, the system will tend to minimize step length, and clusters are unstable: they expand or shrink, depending on the precise kinetics and environment. As an example, an assembly of clusters will coarsen due to Ostwald 20 or coalescence ("Smoluchowski")21 ripening.

Let $\mathbf{r}_{\mathrm{CM}}$ denote the position of the center of mass of the cluster. We define the mean velocity:

$$
\overline{\mathbf{V}}=\left\langle\partial_{t} \mathbf{r}_{\mathrm{CM}}\right\rangle,
$$

and the non-equilibrium tracer diffusion constant:

$$
D_{c}=\frac{\left\langle\left|\mathbf{r}_{\mathrm{CM}}-\overline{\mathbf{V}} t\right|^{2}\right\rangle}{4 t},
$$

which reduces to the usual equilibrium cluster diffusion constant $D_{c}^{e q}$ when $\overline{\mathbf{V}}=0$. This quantity could also be defined as half the diffusion constant describing the relative motion of two identical islands, whether they are drifting or pat. This latter definition has been useful experimentally 22 Shape fluctuations of the cluster are measured by the island roughness

$$
W^{2} \equiv\left\langle R^{2}\right\rangle-\langle R\rangle^{2} .
$$

At equilibrium, using Eq. (14), we find:

$$
W_{e q}^{2}=\frac{3 k_{B} T R_{0}}{4 \tilde{\beta}} .
$$

As a static property, $W_{e q}^{2}$ does not depend on the transport mechanism, just like the static spectrum in Eq.14.

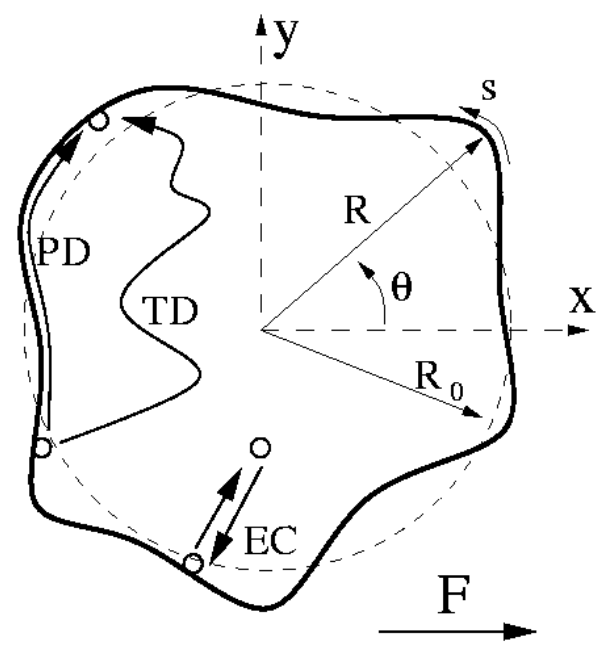

FIG. 1. Three processes are involved in mass transport: Diffusion along the step, diffusion across the terraces, and attachment-detachment of adatoms at the step. When one of these processes is slower than the others, we get the PD, $\mathrm{TD}$, or EC regime, respectively. Electromigration force $F$ is taken to be along the $x$ axis in the text. $R$ is the distance from the center of the island, $\theta$ is the polar angle with respect to the $x$ axis, and $s$ is the arclength along the step. $R_{0}$ is the mean radius.

A major goal of our analysis is to explore properties of driven clusters on the dominant mechanism of mass transport. A crude criterion for being in the PD regime follows from checking wether the diffusion constant is dominated by line diffusion or diffusion across 
the terrace. Comparing expressions (25) and (34) for $D_{c}^{e q}$ given below, one finds: $R_{0}^{2} \ll\left(R_{0}+d\right) \ell_{c}$, where $d=d_{+}$or $d_{-}$and $\ell_{c} \equiv D_{L} / D a c_{e q}^{0} 23$ The opposite case, $R_{0}^{2} \gg\left(R_{0}+d\right) \ell_{c}$, will be denoted as the $2 \mathrm{D}$ transport regime. In this regime, TD and EC limits are defined by $R_{0} \gg d_{+}$or $d_{-}$, and $R_{0} \ll d_{+}$and $d_{-}$, respectively. Note that the cluster radius $R_{0}$ is involved in these relations: it is a geometrical cut-off for long-wavelength fluctuations. A more general definition of these regimes involving wavelength dependence can be found in Ref. 13.

\section{PD LIMIT}

\section{A. Steady states and stability analysis}

Some known results about 2D voids in metal electric line can be transposed directly to the case of vacancy islands in the PD limit. Best known is "Ho's law," which states that the drift velocity varies inversely with the radius. seen in recent Monte Carlo simulations of electromigration on $\mathrm{Cu}\{001\}$ using semi-empirical energy barriers.25 In our formalism, the drift velocity (in the $x$-direction) of a $2 \mathrm{D}$ cluster with mean radius $R_{0}$ is:

$$
\bar{V}=\phi \frac{a D_{L}}{\xi_{s t} R_{0}},
$$

$\phi=1(-1)$ denotes atom (vacancy) islands. Thus, atom islands and vacancy clusters drift in opposite directions. With current densities about $10^{4}-10^{6} \mathrm{~A} \mathrm{~cm}^{-2}$ at 600 $\mathrm{K}$ on metals, we find $\xi_{s t} \approx 10^{5} \AA$. Assuming next an activation barrier of $\sim 0.5 \mathrm{eV}$ along the periphery and a concentration of mobile atoms per site $c_{s t} \approx 0.1$, we estimate $D_{L} \approx 10^{9} \AA^{2} / \mathrm{s}$; thus, for an island of radius $10^{3} \AA, \bar{V} \approx 10 \AA / \mathrm{s}$.
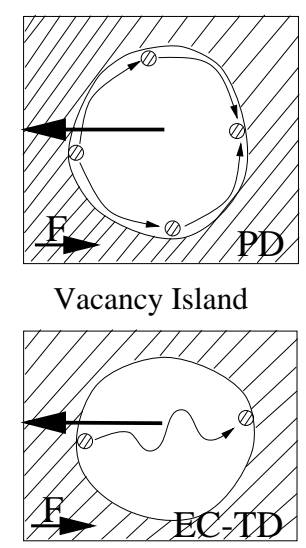

FIG. 2. Drift direction of island as a function of mass transport mechanism. We have assumed a direct Ehrlich-Schwoebel effect.
Stability analysis for void 26,27 can also be applied to our case. Since we assume isotropic step properties, the equilibrium shape of the island is circular. With increasing electromigration, this steady-state shape persists so long as the island is stable. A hand-waving argument allows us to retrieve the instability threshold found in Refs. 26 and 27 . We simply have to compare fluxes along the step close to a protrusion of the step inside the cluster. The flux contributing to destabilization is $J_{\text {destab }} \sim D_{L} / \xi$. Stabilizing effects related to line tension effect result in a flux $J_{\text {stab }} \sim D_{L} \Gamma / R_{0}^{2}$, where curvature changes are taken to be $\sim 1 / R_{0}$ between points separated by a distance $\sim R_{0}$. The protrusion increases if $J_{\text {destab }}>J_{\text {stab }}$. The instability criterion is then $|\chi|>\chi_{c}$, where $\chi \equiv R_{0}^{2} / \Gamma \xi=\left(F_{\mathrm{em}} / \tilde{\beta}\right)\left(R_{0} / \mathrm{m}^{2}\right)^{2}$ According to a linear stability analysis, $\chi_{c}=10.65$. 26 , 27 With the preceding parameters and $\tilde{\beta}=0.3 \mathrm{eV} / a$, we find that islands with radius larger than $R_{c} \approx 5 \cdot 10^{3} \AA$ will be unstable. The instability appears at a characteristic time $\tau=\chi_{c} \Gamma \xi^{2} /\left(a D_{L}\right) \approx 10^{2}$ s. Thus, this phenomenon should be observable experimentally. The instability criterion is the same for atom and vacancy islands.

Since electromigration of single-layer clusters is unaffected by their shape, our voids can be characterized as conducting. In contrast, voids in electric lines are essentially insulating. The resulting current-crowding effects qualitatively change the nature of the instability: the void is linearly stable, but becomes unstable under finite perturbations. At late stages of this instability, the void splits.28

\section{B. Diffusion constant and fluctuations}

Keeping only the line diffusion term in Eq. (10), one gets in the frame moving with the mean velocity $\bar{V}$ of the island:

$$
\begin{aligned}
& V_{n}-\bar{V} n_{x}=-\partial_{s}\left[J_{s t}\right] \\
= & -\partial_{s}\left[\frac{a}{\xi_{s t}} D_{L} \partial_{s}[R(\theta) \cos (\theta)]-a D_{L} \partial_{s}(\Gamma \kappa)-q_{s t}\right],
\end{aligned}
$$

where $n_{x}$ is the $x$ component of the unit vector $\mathbf{n}$ normal to the step. The normal step velocity is written as:

$$
V_{n}=\phi \frac{\partial_{t} R}{\left[1+\left(\partial_{\theta} R / R\right)^{2}\right]^{1 / 2}},
$$

and the step curvature is defined with the sign convention:

$$
\kappa=\phi \frac{R^{-1}+\partial_{\theta \theta} R^{-1}}{\left[1+\left(\partial_{\theta} R / R\right)^{2}\right]^{3 / 2}} .
$$

We then linearize Eq. (20) for small deformation of the island $\rho(\theta)$. With the Fourier transform of $\rho(\theta, t)$ as defined by Eq. (13), Eq. (20) takes the form: 


$$
\begin{aligned}
& {\left[i \omega \tau+n^{2}\left(n^{2}-1\right)\right] \rho_{n \omega}} \\
& -n\left[(n+2) \rho_{n+1, \omega}+(n-2) \rho_{n-1, \omega}\right] \phi \chi=\frac{i n \tau}{R_{0}} \phi q_{n \omega},
\end{aligned}
$$

where $\tau=a D_{L} \Gamma / R_{0}^{4}$. This equation implies that $\rho_{0}$ is a constant; but this constant must vanish $\left(\rho_{0}=0\right)$ since $\rho$ is defined as the departure from the mean radius $R_{0}$. In Eq. (23) the eigenvalues $i \omega$ do not depend on the sign of $\chi$ (as expected physically). Thus, these eigenvalues also do not depend on $\phi$. Hence, as stated above, atom and vacancy islands have the same instability criterion $|\chi|>\chi_{c}$.

We first consider the case $\chi \ll 1$. We then expand step fluctuations in the form $\rho_{n}=\rho_{n}^{(0)}+\chi \rho_{n}^{(1)}+\chi^{2} \rho_{n}^{(2)}+\ldots$. To 0th order, we simply get Eq. (23) without the term proportional to $\chi$. The diffusion constant is calculated using the Fourier space relation:

$$
D_{c}=\left\langle\left|\rho_{1}\right|^{2}\right\rangle / t .
$$

The equilibrium diffusion constant reads:

$$
D_{c}^{e q}=\frac{a^{3} D_{L}}{\pi R_{0}^{3}} .
$$

To higher orders in $\chi$, Eq. (23) shows the Langevin force $q$ does not explicitly intervene, ultimately because $D_{L}$ is supposed to be independent of electromigration; hence,

$$
\begin{aligned}
& {\left[i \omega \tau+n^{2}\left(n^{2}-1\right)\right] \rho_{n \omega}^{(m+1)}} \\
& =n\left[(n+2) \rho_{n+1, \omega}^{(m)}+(n-2) \rho_{n-1, \omega}^{(m)}\right] \phi \chi
\end{aligned}
$$

Using this relation, we calculate the first correction to the cluster diffusion constant:

$$
D_{c}=D_{c}^{e q}\left(1+\frac{\chi^{2}}{4}+O\left(\chi^{4}\right)\right) .
$$

The non-equilibrium cluster roughness can be calculated in a similar way. We find:

$$
W^{2}=W_{e q}^{2}\left(1+b \chi^{2}+O\left(\chi^{4}\right)\right),
$$

where $b \simeq 0.23$ is a numerical constant resulting from infinite summations. Since $\chi$ is of order unity, this correction can be non-negligible. Note that the first correction to these quantities is proportional to the electromigration force squared. Correspondingly, $W$ and $D_{c}$ are invariant under the inversion symmetry $F \rightarrow-F$.

Fluctuations increase with $F$. In a linear theory, they should diverge at the instability threshold. If we define $\epsilon \equiv \chi_{c}-\chi$, then close to the threshold, Eq. (23) can be written in terms of the vector $\vec{\rho}=\left\{\rho_{n}:|n| \geq 2\right\}$

$$
\mathbf{I} i \omega \vec{\rho}=\mathbf{M} \vec{\rho}+\vec{b},
$$

where $\vec{b}$ is a noise term, $\mathbf{I}$ is the identity matrix and the matrix $\mathbf{M}$ is expanded as $\mathbf{M}=\mathbf{M}_{\mathbf{c}}+\epsilon \mathbf{M}_{\mathbf{1}}$, where $\mathbf{M}_{\mathbf{c}}$ has one null eigenvalue (as can be seen, for example, in Ref.27, table II). It is then easily shown that the squared roughness

$$
\begin{aligned}
W^{2} & =\left\langle\vec{\rho}^{T} \vec{\rho}\right\rangle \\
& =\int \frac{d \omega}{2 \pi}\left\langle\vec{b}^{T}(i \omega \mathbf{I}+\mathbf{M})^{-1 T}(-i \omega \mathbf{I}+\mathbf{M})^{-1} \vec{b}\right\rangle
\end{aligned}
$$

diverges like $\epsilon^{-1}$ when $\epsilon \rightarrow 0$. Hence, approaching the instability threshold,

$$
W \sim \epsilon^{-1 / 2} .
$$

Note that $D_{c}$ does not diverge: the instability concerns the morphology of the island, not its motion. The divergence of $W$ is analogous to the case of an isolated step subject to a morphological instability during growth,29 where the same exponent was found. Close to threshold, fluctuations become large, and nonlinear effects should be addressed, as shown in Ref. 30 during growth.

\section{Monte Carlo simulations}

We here use kinetic Monte Carlo simulations to show that the morphological instability of an island under periphery diffusion leads to splitting.

We perform a 2D Monte Carlo simulation on a square lattice. (Equivalently, the simulation can be described as an SOS model in which the height of the surface is either 0 or 1.) The energy barrier for a move is taken to be proportional to the number of in-plane nearest $(n)$ and next-nearest $\left(n^{\prime}\right)$ neighbors of the atom before hopping. Next-nearest neighbors are included to reduce the anisotropy of the steps. Moves leading to an adatom (i.e. an atom with no occupied nearest or next-nearest neighbor sites) are forbidden. Atoms are allowed to hop only to nearest-neighbor sites. Electromigration is taken into account as a direction-dependent bias in the hopping barriers. The total barrier energy is then written:

$$
E_{i j k}=\varepsilon_{\mathrm{d}}\left(n_{i j}+n_{i j}^{\prime}\right)+\varepsilon_{\mathrm{em}} \cos \left([k-1] \pi / 2-\theta_{0}\right),
$$

where $i$ and $j$ are position coordinates along the $x$ and $y$ axis, respectively; $n_{i j}$ and $n_{i j}^{\prime}$ are the number of nearest and next-nearest neighbors, respectively; $k=1,2,3,4$ is the direction of the move (to one of the nearest neighbors); and $\varepsilon_{\mathrm{d}}$ and $\varepsilon_{\mathrm{em}}$ are the energies associated with diffusion and electromigration, respectively. Explicitly, $\varepsilon_{\mathrm{em}}=F_{\mathrm{st}} a / 2 ; \theta_{0}$ is the angle between of electromigration force and the [10] axis of the lattice. Atoms and moves are picked randomly, and a move is performed with probability proportional to $\exp \left(-E_{i j k} / k_{B} T\right)$.

A circular vacancy is chosen as the initial state. The late stage of the inctability has been the subject of a recent controversy 2628 As for insulating voids in Ref. 28, our atom and vacancy islands (equivalent to conducting voids) split when they are unstable, as illustrated in 
Fig. 3b. For weaker electromigration (i.e. smaller $\chi$ ), the cluster is stable, as shown in Fig. 3a. In this figure, the steady state of the cluster at long time is slightly elongated along the electromigration axis. This elongation is probably a consequence of the anisotropy of $D_{L}$ or $\tilde{\beta}$, which is not completely avoided in the simulations.

Quantitatively the instability appears for radii larger than $R_{c} \approx 13 a$ at $k_{B} T=0.6 \varepsilon$, where the characteristic energy $\varepsilon$ is the energy of a single bond. We note that an elementary kink costs energy $\varepsilon$ in our model. In the "restricted" approximation, in which only elementary kinks (i.e. those repositioning the step by a single row) are allowed, the step stiffness becomes:

$$
\tilde{\beta}=\left(2+e^{\varepsilon / k_{B} T}\right) \frac{k_{B} T}{2 a} .
$$

Using this relation, we find $\tilde{\beta}=2.2 \varepsilon / a$. Now from the criterion $|\chi|>\chi_{c}$, we get $R_{0}>R_{c} \approx 10.8 a$, in good agreement with our simulations. Note that the instability can also be used to determine $\tilde{\beta}$ or $F$ when the other parameters are known. From our simulation, with $R_{c}=13 a$, we find $\tilde{\beta}=3.3 \varepsilon / a$.

As expected from the model, the same instability is found for atom islands.

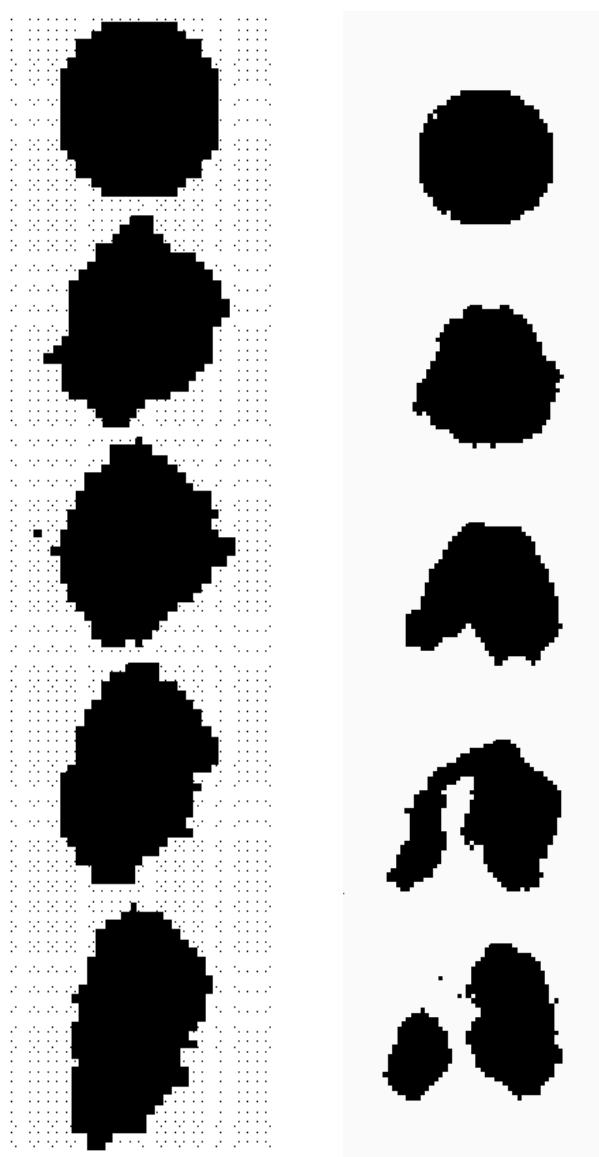

FIG. 3. Monte Carlo simulations of a vacancy island in the PD limit, with $F=0.1 \varepsilon / a$ and $k_{B} T=0.6 \varepsilon$. a) Stable island, at $R_{0}=10 a$. b) Splitting, at $R_{0}=15 a$.

\section{2D TRANSPORT REGIME}

\section{A. Steady states}

In the opposite limit of 2D transport, the equilibrium behavior of these islands is well known.13 The cluster diffusion constant is

$$
D_{c}^{e q}=\frac{a^{4} D c_{e q}^{0}}{\pi R_{0}}\left(\frac{1}{R_{0}+d_{+}}+\frac{1}{R_{0}+d_{-}}\right) .
$$

The corresponding scaling expectation of Eq. (3) is confirmed readily for weak electromigration, where we can work to first order in $\xi^{-1}$. The islands remain circular and drift with velocity

$$
\bar{V}=-\frac{a^{2} D c_{e q}^{0}}{\xi}\left(\frac{R_{0}}{R_{0}+d_{+}}-\frac{R_{0}}{R_{0}+d_{-}}\right) .
$$

The first conclusion is that an Ehrlich-Schwoebel effect $\left(d_{-}>d_{+}\right)$is needed for non-zero drift. We note in particular the remarkable prediction that for EC, the drift velocity actually increases with increasing island size. This behavior has been seen in Monte Carlo simulations by Wickham and Sethna, 1 and leads to an exponential coarsening of an assembly of clusters when coalescence is induced by drift-velocity differences. Eq. (35) allows us to evaluate $d_{+}$inside the voids of Ref 4 . Their findings lead to the expected result that the length $d_{+}$increases as temperatures decreases. Moreover, their phenomenological fitting expression for asymptotically large islands (cf. the caption of their Fig. 6), $|\bar{V}|=v_{0}-v_{1}\left(a / R_{0}\right)$, can be retrieved from an expansion of Eq. (35) for $R_{0} \gg d_{+}$ (and $d_{-} \rightarrow \infty$ ). Thus, we can identify their coefficients: $v_{0}=a^{2} D c_{e q}^{0} / \xi$ and $v_{1}=v_{0} d_{+} / a$.

Since Eq. (35) does not depend on $\phi$, both atom and vacancy islands drift in the same direction in the $2 \mathrm{D}$ transport regime. Thus, we obtain a very simple way to identify the dominant mass transport mechanism, since atom and vacancy islands drift in opposite directions in the PD limit. (Cf. Eq. (19).) Note also that when $d_{-}>$ $d_{+}$- expected for the typical Ehrlich-Schwoebel effect - the direction of motion of the (adatom or vacancy) cluster in the 2D transport regime is opposite to the electromigration force. (Cf. Fig. 2.)

For a vacancy or an atom cluster in the TD limit, the circular steady state is an exact solution of the equations of motion (even in the nonquasistatic limit). As soon as the attachment is not instantaneous $\left(d_{+} \neq 0\right)$, this is no longer true. To second order in $\xi^{-1}$, a non-circular shape is found in steady state for the "interior" model (e.g. appropriate to a vacancy island with an infinite Ehrlich-Schwoebel barrier) when $d_{+} \neq 0$. This shape is elongated perpendicular to the electromigration force for vacancy islands (along the electromigration force for atom islands), as can be understood from an intuitive argument: For small sticking probability, atoms detaching from the sides of the vacancy island, will drift along 
the electromigration axis, but will also have a residual drift toward the center of the vacancy if they attach only after several attempts.

A macroscopic description, analogous to the Bernoulli effect, provides an intuitive understanding of this phenomenon. According to this effect, the pressure variation perpendicularly to a flow is proportional to the kinetic energy involved in this flow. Considering the flow of adatoms inside the island, this relation takes the form:

$$
J_{\text {att }} \times m V_{a t t} \propto \frac{1}{2} m c_{e q} V_{a d a t}^{2},
$$

where the l.h.s. is the pressure variation and the r.h.s. is the kinetic energy of the flow. The adatom mass divides out, it is irrelevant here because the dynamics is overdamped. The first factor on the l.h.s.,

$$
J_{a t t}=\nu_{+}\left(c-c_{e q}\right)
$$

is the flux of atoms attaching to the steps. The second factor $m V_{a t t}$ is the momentum associated with atoms attaching to the step, i.e., the product of the adatom mass $m$ and the macroscopic velocity

$$
V_{a t t}=D /\left(R_{0}+d_{+}\right)
$$

associated with motion of an adatom across the island during its lifetime. On the r.h.s. of Eq. (36), we use $V_{a d a t}$, defined as the adatom drift velocity needed to move the step at velocity $\bar{V}$. Hence,

$$
V_{a d a t} c_{e q}=\bar{V} / a^{2},
$$

where the cluster velocity $\bar{V}$ is given by Eq. (35) As a macroscopic property, $V_{a d a t}$ is different from the drift velocity $\langle v\rangle$ of one adatom as defined in Eq. (11). Using Eqs. (37-39) in the Bernoulli relation Eq. (36), we get the effective concentration change induced by the electromigration force:

$$
c-c_{e q} \propto c_{e q} \frac{R_{0}^{2}}{\xi^{2}} \frac{d_{+}}{R_{0}+d_{+}} .
$$

Moreover, the concentration variation associated with a change of local radius is known from Gibbs-Thomson relation

$$
c-c_{e q} \propto c_{e q} \Gamma \delta R / R_{0}^{2} .
$$

Balancing the destabilizing effect in Eq. (40) to the stabilizing one in Eq. (41), we get a condition for a steadystate:

$$
\frac{\delta R}{R_{0}} \propto \frac{1}{\Gamma} \frac{R_{0}^{2}}{\xi^{2}} \frac{d_{+} R_{0}}{d_{+}+R_{0}} .
$$

Expanding our interior model to second order in $1 / \xi$, the island deformation involves only the $n=2$ mode. Defining

$$
\Delta \equiv \frac{1}{2 R_{0}}[\rho(\pi / 2)+\rho(-\pi / 2)-\rho(0)-\rho(\pi)],
$$

we find, quantitatively,

$$
\Delta=\frac{1}{\Gamma} \frac{R_{0}^{2}}{6 \xi^{2}} \frac{d_{+} R_{0}}{d_{+}+R_{0}},
$$

in agreement with the evaluation in Eq. (42) based on the Bernoulli effect. In the EC limit, with $R_{0} \sim 10^{3} a$ and $\xi \sim 10^{5} a$, one finds that $\Delta \sim 10^{-2}$. Thus, the deformation is small for an electromigrating island with interior dynamics.

When mass exchange with the exterior is allowed, there is no steady state. To investigate further, we study the case of an (atom) island exchanging matter only with the exterior, but with desorption allowed. A term $F_{e q}-c / \tau_{s}$ is added to Eq. (6). Although the island is not stable in this case, we further consider its behavior in order to analyze the tendency to deviate from circularity as it shrinks. To first order in $1 / \xi$ we find a circular steady state with velocity

$$
\bar{V}=-\frac{1}{\xi} \Omega D c_{e q} \frac{2 x_{s} K_{1}}{2 x_{s} K_{1}+d_{+}\left(K_{2}+K_{0}\right)}
$$

where $K_{n} \equiv K_{n}\left(R_{0} / x_{s}\right)$ is the modified Bessel $K$ function and $x_{s}=\left(D \tau_{s}\right)^{1 / 2}$. The $n=0$ mode is unstable, and the island has a characteristic decay time

$$
\tau_{\text {decay }}=\frac{4 \pi R_{0}^{2}}{\Omega D c_{e q}} \frac{x_{s} K_{0}+d_{+} K_{1}}{K_{1}} .
$$

To second order in $\xi^{-1}$ the island shape is not circular anymore, and the resulting deformation reads:

$\Delta=-\frac{d_{+}}{3 \Gamma} \frac{R_{0} x_{s}}{8 \xi^{2}} \frac{2 x_{s}}{K_{1}+K_{3}} \frac{R_{0}\left(K_{2}-K_{1} K_{3}\right)-x_{s} K_{2} K_{1}}{x_{s} K_{1}+d_{+}\left(K_{0}+K_{2}\right) / 2}$

Since $\Delta$ is negative, the elongation of an atom island is now along the electromigration axis.

This can also be qualitatively understood from the Bernoulli effect, and the origin of elongation along the electromigration axis can be traced back to a change of sign of the curvature in the Gibbs-Thomson relation (41). To adapt the local equilibrium at the step to the lowering of the pressure on the sides of the island, the curvature has to be locally decreased, so that the island elongates along the electromigration axis. In the limit of small desorption, we get:

$$
\Delta=-\frac{1}{2 \Gamma} \frac{R_{0}^{2}}{\xi^{2}} \frac{x_{s} d_{+}}{R_{0}+d_{+}}
$$

Note that $\Delta$ diverges when $x_{s} \rightarrow \infty$, in agreement with the previous claim that there is no steady state in this limit. We notice that this formula is similar to Eq. (44), with a factor of $R_{0}$ replaced by the new cut-off length $x_{s}$. Taking $\Gamma \sim a, d_{+} \sim 10^{4} a, R_{0} \sim 10^{3} a, x_{s} \sim 10^{6} a$, and $\xi \sim 10^{8} a$, we get $\Delta \sim 0.5$. Hence, a large deformation of the island can be found in the exterior model. 


\section{B. Validity of the quasistatic limit}

In this section, we consider a vacancy island in the absence of electromigration and in the non-quasistatic regime. We use polar coordinates $r$ and $\theta$. The $n$th component of the Fourier transform of the concentration with respect to $\theta$ is expanded as

$$
c_{n}(r, t)=\sum_{m=0}^{\infty} a_{n m}(t) r^{m}
$$

Using this expression in the interior model, we look for solutions of the form $a_{n m}(t)=\exp (i \omega t) a_{n m}$. By symmetry $a_{n m}=a_{-n m}$, and from analyticity of $c$ at $r=0$, $a_{n m}=0$ when $n-m$ is odd. Using the recursion equations resulting from Eqs.(4), it is the possible to show that $a_{n m}=0$ when $m \leq|n|$, and that all $a_{n m}$ can be calculated from the free parameters $a_{n|n|}$. We thus calculate the $a_{n m}$ as a function of the $a_{n|n|}$, and plug this result into the boundary conditions Eqs. (7710) -with $J_{s t}=0$. We find the non-quasistatic dispersion relation from the requirement that the prefactors of the $a_{n|n|}$ 's should cancel:

$$
\begin{aligned}
\lambda & =-a^{2} \tilde{c}_{e q} \frac{\Gamma}{R_{0}}\left(n^{2}-1\right) \times \\
& \times\left[\frac{d_{+}}{R_{0}}+\left(|n|+\lambda^{1 / 2} \frac{I_{|n|+1}\left(\lambda^{1 / 2}\right)}{I_{|n|}\left(\lambda^{1 / 2}\right)}\right)^{-1}\right]^{-1},
\end{aligned}
$$

where $I_{n}$ is the modified Bessel $I$ function, and $\tilde{c}_{e q}=$ $c_{e q}^{0} \exp \left(-\Gamma / R_{0}\right)$. Since $\lambda \equiv i \omega R_{0}^{2} / D, \Re e[\lambda]$ is proportional to the growth rate of the perturbations. In the quasistatic limit, Eq. (6) is solved on the interior terrace instead of Eq. (4). We then find the following dipersion relation:

$$
\lambda=-a^{2} \tilde{c}_{e q} \frac{\Gamma}{R_{0}}\left(n^{2}-1\right)\left[\frac{d_{+}}{R_{0}}+|n|^{-1}\right]^{-1}
$$

Note that in the quasistatic limit, the $n=0$ mode is frozen (i.e. $\lambda=0$ when $n=0$ ). Moreover, we obtain here the standard TD (or EC) limit by letting $d_{+} \rightarrow 0$ (or $d_{+} \rightarrow \infty$ ), leading to $i \omega \sim R_{0}^{-2}$ (or $\sim R_{0}^{-3}$ ). From Eq. (50), the quasistatic limit Eq. (51) is recovered when $|\lambda| \ll n^{2}$. Using the quasistatic expression for $\lambda$, this condition can be rewritten:

$$
\Gamma a^{2} \tilde{c}_{e q} \nu_{+} \ll \frac{R_{0} \nu_{+}}{|n|}+D,
$$

where $|n|>1$. For the approximation to be valid for all modes, it is sufficient to satisfy the stronger condition

$$
\Gamma a^{2} \tilde{c}_{e q} \nu_{+} \ll D
$$

This inequality should be interpreted as follows: the effective diffusion constant of the step (l.h.s.) must be much smaller than that of the adatoms on the terrace (r.h.s.).

The mode-dependent term on the r.h.s. of Eq. (52), related to attachment-detachment, indicates that the quasistatic limit is more difficult to achieve at large $|n|$, i.e. small wavelengths, because these modes have shorter relaxation times. Using a small-wavelength cutoff $|n|^{*} \sim R_{0} / a$ on the r.h.s. of Eq. (52), we see that Eq. (53) is valid in the limit of slow kinetics, $\nu_{+} \ll D / a$. Conversely, when $\nu_{+} \gg D / a$, we find

$$
a \Gamma \tilde{c}_{e q} \ll 1,
$$

which is the criterion for the quasistatic limit to be valid in the fast-kinetics (i.e. TD) regime.

As an example, let us consider the case of $\mathrm{Si}(111)$ at $T \sim 1000 \mathrm{~K}$. From Ref. 31, we have $a^{2} c_{e q}<0.1$ and $d_{+}=D / \nu_{+} \sim 10^{4} a$, and from Ref. 32, $\Gamma \sim 10 \AA$. Eq. (53) is then easily checked to be valid: the quasistatic approximation can be used.

\section{Stability analysis}

We now go back to the electromigration problem, and perform a linear stability analysis of the non-quasistatic model equations. We consider the TD limit $\left(d_{+}=0\right)$ of the interior model. We perform a stability analysis of perturbations with respect to the steady state with constant concentration $c=\tilde{c}_{e q}$ and circular shape $R=$ $R_{0}$. To do so, we again expand the concentration using Eq. (49), now in Eqs. (45), and seek solutions of the form $a_{n m}(t)=\exp (i \omega t) a_{n m}$. Matching coefficients for for each $r^{m}$ leads to a set of recursion equations for the $a_{n m}$ 's for $m \geq 2$ :

$$
\begin{aligned}
\frac{i \omega}{D} a_{n, m-2}=-a_{n m}\left(n^{2}-m^{2}\right) & -\frac{1}{2 \xi}\left[(n+m) a_{n+1, m+1}\right. \\
& \left.-(n-m) a_{n-1, m-1}\right],
\end{aligned}
$$

supplemented by the relations

$$
\begin{aligned}
& a_{n 0}=0 \text { for } n \neq 0, \\
& a_{n 1}=0 \text { for }|n| \neq 1 .
\end{aligned}
$$

Boundary conditions Eqs. (7 10 ) then provide two set of relations for the $a_{n m}$ 's and the $\rho_{n}$ 's. When the $\rho_{n}$ 's are eliminated, a set of relations among the $a_{n m}$ 's is found:

$$
\begin{array}{r}
0=\sum_{m=0}^{\infty}\left[-\frac{i \omega R_{0}^{2}}{D} a_{n m}-\frac{a^{2} \tilde{c}_{e q}}{R_{0}}\left(n^{2}-1\right) \Gamma\left\{m a_{n m}\right.\right. \\
\left.\left.-\frac{R_{0}}{2 \xi}\left(a_{n+1, m}+a_{n-1, m}\right)\right\}\right] .
\end{array}
$$

Numerical solution of this set of equations reveals an instability for $R_{0} / \xi>0.1$ related to the existence of a positive eigenvalue $i \omega$ in this range of parameters. It is 
interesting to note that the instability threshold is independent of $\Gamma$. To investigate the nature of the instabilities of these islands, we again perform Monte Carlo simulations. Rather than the splitting seen in the PD limit, we find a new type of instability.

\section{Monte Carlo Simulations}

The code used here is similar to that used for a vacancy island in the PD limit, but with adatoms now allowed inside. Moreover, in order to tune attachment-detachment kinetics, the hopping probability is decreased by a factor $p, 0<p \leq 1$ when the number of neighbors changes during the hop. 33

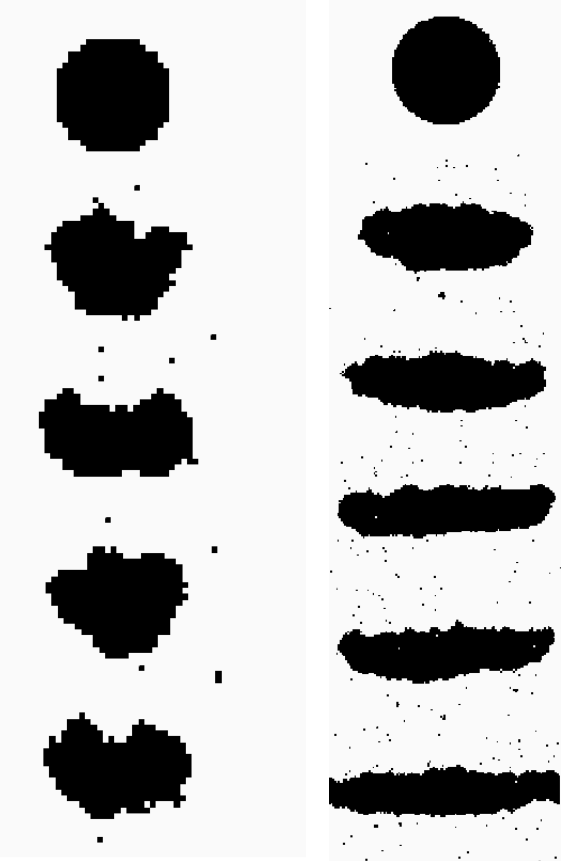

FIG. 4. Monte Carlo simulations of a vacancy island in the TD limit, with $F=0.1 \varepsilon / a, k_{B} T=0.6 \varepsilon$. a) "Stable" island, at $R_{0}=10 a$. b) Slit formation, for $R_{0}=30 a$.

At high temperature $k_{B} T \geq 0.7 \varepsilon$, micro-vacancies escape noticeably from the steps. This might heal possible instabilities by producing a micro-vacancy where a cusp is present. We therefore chose to perform our simulations at $k_{B} T=0.6 \varepsilon$. Even though a few micro-vacancies still escape from the clusters at this temperature, they are rare enough so that their effect on the dynamics can be considered negligible.

No drastic change in the island shape is seen at the threshold given by the linear stability analysis. Let us define the front and the back side of the island with respect to cluster motion as the first and the last part passing at a given value of $x$. When electromigration is increased, the front side is stabilized. The back side is destabilized and exhibits chaotic behavior, but its roughness remains finite, as shown in Fig. 4a. We conjecture that this is the instability that we found from linear analysis. Although some instability is seen in this regime, we classify the island as stable because its shape is not affected drastically.

Increasing the electromigration force, we see that unstable slit-like shapes appear, as depicted in Fig. 4 b. Since these shapes are seen for all orientations of the electromigration force, they are not due to lattice anisotropy. This instability contrasts qualitatively with the splitting found in the PD regime (see Fig. $3 \mathrm{~b}$ ).

Changing the value of $p$ does not seem to change this scenario, but we have not performed simulations in the limit where $p$ is small $(p<0.1)$ due to the large computation time needed there. Numerical solution of the model equations would be needed to understand in greater detail these different regimes.

\section{ELECTROMIGRATION BIAS IN ATTACHMENT-DETACHMENT}

Finally, we introduce a possible electro-bias between attachment and detachment. Extracting one atom from the step, not counting any bond breaking, requires work $a \mathbf{F}_{\mathrm{em}} \cdot \hat{\mathbf{n}}$ along the normal to the step. 34 From a linearized Gibbs-Thomson relation, we find that the effective equilibrium concentration becomes

$$
c_{e q}=c_{e q}^{0}\left(1+a \mathbf{F}_{\mathrm{em}} \cdot \hat{\mathbf{n}} / k_{B} T\right) .
$$

Defining $\xi_{ \pm}^{-1}=\mathbf{F}_{\mathrm{em}} \cdot \hat{\mathbf{n}} / k_{B} T$ on the lower or upper side of the boundary step edge, respectively, we find that electro-bias contributes to the velocity of Eq. (35) an extra term

$$
\delta \bar{V}=a^{3} D c_{e q}^{0}\left(\frac{\xi_{1,+}^{-1}}{R_{0}+d_{+}}-\frac{\xi_{1,-}^{-1}}{R_{0}+d_{-}}\right),
$$

where the subscript indicates the $n=1$ component of the Fourier transform of $1 / \xi_{ \pm}(\theta)$. When there is no EhrlichSchwoebel effect $\left(d_{+}=d_{-}\right), \bar{V}$ from Eq. (35) vanishes, and the drift velocity is only due to this new contribution combined with PD effects. In the EC limit, $\delta \bar{V}$ does not depend on the size of the cluster, while in the TD limit $\delta \bar{V} \sim 1 / R_{0}$. This is the same size dependence as that associated with the PD limit. Since $\delta \bar{V}$ decreases with increasing island size, we expect this contribution to be small for large islands.

The deformation of the cluster is proportional to $1 / \xi$, rather than $1 / \xi^{2}$ as seen in the other regimes. Thus electrobias might induce larger deformations of the clusters. However, since we do not know the angular dependence of these forces, we cannot go further in the analysis.

\section{SUMMARY AND PERSPECTIVES}

We have studied the response of single-layer clusters to electromigration in different regimes associated with 
different mass-transport mechanisms. Some of the main results are collected in table 1 . The drift velocity and shape changes agree with those found in earlier published calculations, 2 and give a theoretical explanation and explicit expressions for those found with Monte Carlo simulations.

The instability is found to depend qualititatively on the mass transport mechanism. PD is associated with splitting, and 2D transport with slit formation. Although the instability threshold of the latter may be beyond the experimental range for $2 \mathrm{D}$ islands, it could well be important in the case of disorderefoxygen domains in $\mathrm{YBaCuO}$, 3.4 and for void dynamics, 26 28 where higher current densities are used. In the latter case, 2D transport might occur on the substrate across the void.

Fluctuations are found to be affected by electromigration. The non-equilibrium cluster diffusion constant and roughness were studied. The island roughness is found to diverge as the instability threshold is approached.

Non-linear analysis is needed for a better understanding of these instabilities. Moreover, cross-over regimes should be studied in order to catalogue completely the behavior of these islands. For a more realistic description of the islands, especially at temperature, anisotropy should also be included.4.28,35 Indeed, it was already shown experimentally that extremely anisotropic properties (such as diffusion) could lead to very different behavior 36

\begin{tabular}{|c|c|c|c|}
\hline & $P D$ & $T D$ & $E C(A D)$ \\
\hline $\begin{array}{l}\text { Length } \\
\text { criterion }\end{array}$ & $R_{0}^{2} \ll \ell_{c}\left(R_{0}+d\right)$ & $R_{0} \gg d_{+}, d_{-}$ & $R_{0} \ll d_{+}, d_{-}$ \\
\hline$\left\langle V\left(R_{0}\right)\right\rangle$ & $1 / R_{0}$ & 1 & $R_{0}$ \\
\hline $\begin{array}{l}\text { Atom/vacancy } \\
\text { direction }\end{array}$ & Opposite & Sar & \\
\hline Steady state & \multicolumn{2}{|c|}{ Circular } & $\begin{array}{c}\text { None } \\
\text { Non-circ. }\end{array}$ \\
\hline Instability & \multirow{2}{*}{ Splitting } & \multirow{2}{*}{\multicolumn{2}{|c|}{$\begin{array}{l}\text { "Slit" } \| \mathbf{F} \\
\text { Slit } \perp \mathbf{F}\end{array}$}} \\
\hline morphology & & & \\
\hline$D_{c}$ & $D_{c}^{e q}\left(1+\chi^{2} / 4\right)$ & \multicolumn{2}{|c|}{ Anisotropic } \\
\hline
\end{tabular}

TABLE I. Summary of results. In general the entries apply to both atom and vacancy islands. The criteria for the three regimes in terms of the characteristic lengths are given in the first row. The inequality for PD is dominant; when it holds, one has PD even if the inequality for TD or EC is satisfied. The characteristic length $\ell_{c}$ is $D_{L} / a D c_{e q}^{0}$, with equilibrium values for the parameters. For the third row, the relative direction of the drift velocity of atom and vacancy islands is tabulated. In the fourth and fifth rows, the upper (lower) entry is for atom/exterior (vacancy/interior) islands. PD, $\mathrm{TD}$, and EC indicate the mode of mass transport: periphery diffusion, terrace diffusion, and evaporation-condensation, respectively. Reidentifying the letter $\mathrm{D}$, the last is often called $\mathrm{AD}$ (attachment-detachment) to indicate that the process involves just a $2 \mathrm{D}$ "gas" on the terraces.
This study is a step toward the understanding of the evolution of more complex surface geometries in the presence of a diffusion drift, such as 3D islands or groves or assemblies of clusters. To complete this exploration, the fluctuations in the 2D transport regime should be studied. It would also be useful to check with Monte Carlo simulations the predictions about the diffusion constant and the divergence of morphological fluctuations.

We note in closing that, as the surface is affected by electromigration, so electromigration itself is affected by the morphology of the surface. Step shadowing, adatom crowding, or surface roughness induce changes in the local electromigration force. 37 A quantitative description of experiments should take these feedback mechanisms into account.

Aknowledgements: This work was supported by NSF MRSEC grant DMR-96-32521. We acknowledge helpful conversations with E.D. Williams and P.J. Rous, and thank J. Krug for comments on the manuscript.

${ }^{1}$ A.V. Latyshev, A.L. Aseev, A.B. Krasilnikov, and S.I. Stenin, Surf. Sci. 213, 157 (1989).

${ }^{2}$ P.S. Ho, J. Appl. Phys. 41, 64 (1970).

${ }^{3}$ B.H. Moeckly, D.K. Lathrop, and R.A. Buhrman, Phys. Rev. B 47, 400 (1993).

${ }^{4}$ L.K. Wickham and J.P. Sethna, Phys. Rev. B 51, 15017 (1995).

${ }^{5}$ B. Schmittmann and R.K.P. Zia, Statistical Mechanics of Driven Diffusive Systems [Phase Transitions and Critical Phenomena, vol. 17, C. Domb and J.L. Lebowitz, Eds.] (Academic, London, 1995).

${ }^{6}$ G. Rosenfeld, K. Morgenstern, and G. Comsa, in Surface Diffusion: Atomistic and Collective Processes [NATO ASI series B 360], M.C. Tringides, Ed. (Plenum, New York, 1997), 361.

${ }^{7}$ L. Turban, P. Nozières, and M. Gerl, J. Phys. (Paris) 37, 159 (1976); A. Lodder, Sol. St. Comm. 79, 143 (1991).

${ }^{8}$ D. Kandel and E. Kaxiras, Phys. Rev. Lett. 76, 1114 (1996).

${ }^{9}$ H. Ishida, Phys. Rev. B 49, 14610 (1994).

${ }^{10}$ P.J. Rous, T.L. Einstein, and E.D. Williams, Surf. Sci. 315, L995 (1994).

${ }^{11}$ P.J. Rous, Phys. Rev. B 59, 7719 (1999).

${ }^{12}$ E.S. Fu, D.-J. Liu, M.D. Johnson, J.D. Weeks, and E.D. Williams, Surf. Sci. 385, 259 (1997); J.-J. Métois and M. Audiffren, Int. J. Mod. Phys. B 11, 3691 (1997).

13 a) S.V. Khare, N.C. Bartelt, and T.L. Einstein, Phys. Rev. Lett. 75, 2148 (1995); b) S.V. Khare and T.L. Einstein, Phys. Rev. B 54, 11752 (1996); c) 57, 4782 (1998) d) in Dynamics of Crystal Surfaces and Interfaces, P.M. Duxbury and T. Pence, eds. (Plenum, New York, 1997), 83.

${ }^{14}$ S. Stoyanov, Jpn. J. Appl. Phys. 29, L659 (1990).

${ }^{15}$ J.B. Hannon, C. Klünker, M. Giesen, H. Ibach, N.C. 
Bartelt, and J.C. Hamilton, Phys. Rev. Lett. 79, 2506 (1997).

16 J. Heinonen, I. Koponen, J. Merikoski, and T. Ala-Nissila, Phys. Rev. Lett. 82, 2733 (1999).

17 O. Pierre-Louis and C. Misbah, Phys. Rev. Lett. 76, 4761 (1996).

18 J. Villain and A. Pimpinelli, Physique de la Croissance Cristalline, french edition only, (Eyrolles Alea Saclay, Paris, 1995)

${ }^{19}$ In Eq. (6) of ref. 13a and Eq. (17) of ref. $13 \mathrm{~b}$, the numerical factor in the denominator was written as $n^{2}$ rather than $n^{2}-1$. Their results are not affected by this change.

${ }^{20}$ P.W. Voorhees, J. Stat. Phys. 38, 231 (1985); Annu. Rev. Mater. Sci. 22, 197 (1992).

${ }^{21}$ D.S. Sholl and R.T. Skodje, Phys. Rev. Lett. 75, 3158 (1995); C.R. Stoldt, C.J. Jenks, P.A. Thiel, A.M. Cadilhe, and J.W. Evans, J. Chem. Phys. 111, 5157 (1999).

${ }^{22}$ K. Morgenstern, G. Rosenfeld, B. Poelsema, and G. Comsa, Phys. Rev. Lett. 74, 2058 (1995).

${ }^{23}$ In the notation of Ref. $12 \mathrm{~b}, \mathrm{~d}, \ell_{c}=R_{s t}^{2} / R_{s u}$.

${ }^{24}$ E. E. Gruber, J. Appl. Phys. 38, 243 (1967).

${ }^{25}$ H. Mehl, O. Biham, O. Millo, and M. Karimi, Phys. Rev. B 61, 4975 (2000).

${ }^{26}$ M. Mahadevan and R.M. Bradley, J. Appl. Phys. 79, 6840 (1996).

${ }^{27}$ W. Wang, Z. Suo, and T.-H. Hao, J. Appl. Phys. 79, 2394 (1996).

28 M. Schimschak and J. Krug, Phys. Rev. Lett. 80, 1674 (1998); J. Appl. Phys. 87, 695 (2000).

${ }^{29}$ M. Uwaha and Y. Saito, Phys. Rev. Lett. 68, 224 (1992).

${ }^{30}$ A. Karma and C. Misbah, Phys. Rev. Lett. 71, 3810 (1993).

${ }^{31}$ H.-C. Jeong, E.D. Williams, Surf. Sci. Rep. 34, 171 (1999).

${ }^{32}$ C. Alfonso, J.M. Bermond, J.C. Heyraud, and J.J. Métois, J. Phys. I (France), 5, 443 (1995).

${ }^{33}$ D.-J. Liu and J.D. Weeks, Phys. Rev. B 57, 14891 (1998).

${ }^{34}$ For greater accuracy, we should integrate along the detachment path. Note that the force depends on the orientation of the step with respect to $\mathbf{F}$.

${ }^{35}$ M.R. Gungor and D. Maroudas, Appl. Phys. Lett. 72, 3452 (1998).

36 J.-J. Métois, J.-C. Heyraud, and A. Pimpinelli, Surf. Sci. 420, 250 (1999).

${ }^{37}$ P.J. Rous, unpublished. 